\title{
Processo de Desenvolvimento e Avaliação de uma Rede Social Gamificada para conscientização acerca do consumo da Água
}

\author{
Augusto César F. M. Oliveira ${ }^{13}$, Gustavo Henrique F. M. Oliveira ${ }^{23}$, Alisson \\ Pereira da Silva ${ }^{23}$, Máverick André D. Ferreira ${ }^{13}$, Emanoel F. S. Barreiros ${ }^{3}$, \\ Rozelma S. França ${ }^{2}$ \\ ${ }^{1}$ Departamento de Estatística e Informática - Universidade Federal Rural de \\ Pernambuco (UFRPE) \\ CEP 52171 - 900 - Recife - PE - Brazil \\ ${ }^{2}$ Centro de Informática - Universidade Federal de Pernambuco (UFPE) \\ CEP 50.740-560 - Recife - PE - Brazil \\ ${ }^{3}$ Universidade de Pernambuco, Campus Garanhuns (UPE) \\ CEP 55.294-902 - Garanhuns - PE - Brazil \\ \{auguustocesarf, guga.h.f.m.o, alissonnpereira, \\ amaverick70\}@gmail.com, emanoel.barreiros@upe.com, \\ rozelma.soares@gmail. com
}

\begin{abstract}
The discussion on conscious water consumption has become increasingly present in various circles of society. As for the school, being a training institution of citizens, it is up, also the adoption of strategies that seek to educate young people about responsible water consumption. Therefore, this paper describes the process of development and evaluation of a gamified social network geared towards the awareness of responsible water consumption. The evidence in this research indicates positive results for social network with gamified features for the awareness of responsible water use.
\end{abstract}

Resumo. A discussão sobre consumo consciente da água cada vez mais tem se tornado presente nos mais diversos meios da sociedade. À escola, como instituição formadora de cidadãos, cabe, também, a adoção de estratégias que busquem conscientizar os jovens sobre o consumo responsável da água. Logo, o presente trabalho descreve o processo de desenvolvimento e avaliação de uma rede social gamificada voltada a conscientização do consumo responsável da água. As evidências presentes nesta pesquisa, apontam resultados positivos da rede social com características gamificada para a conscientização do uso responsável da água.

\section{Introdução}

A preocupação com o consumo consciente da água tem sido foco de diversas discussões em nível mundial devido à sua importância para a sobrevivência dos seres humanos. Nesse sentido, Jacquiminout (2015) destaca que menos da metade da população 
V Congresso Brasileiro de Informática na Educação (CBIE 2016)

Anais do XXII Workshop de Informática na Escola (WIE 2016)

mundial tem acesso à água potável, sendo distribuído o consumo da água em $73 \%$ para irrigação, $21 \%$ para a indústria e apenas $6 \%$ ao consumo doméstico.

Com base na reflexão dos dados supracitados e no pequeno percentual destinado ao consumo doméstico, aponta-se para a importância do consumo consciente da água. Em 2005, a Assembleia Geral das Nações Unidas instituiu a Década Internacional para Ação: "Água, fonte de vida". No mesmo sentido, o ano de 2013 foi eleito pela UNESCO o "Ano Internacional de Cooperação pela Água", a fim de colocar a temática em pauta com ainda mais expressão (UNESCO, 2013).

Diante disso, a escola, como ambiente de fundamental importância para formação do cidadão, tem a responsabilidade de desenvolver nos jovens as capacidades necessárias, como o pensamento crítico, o aprender a aprender, a tomada de decisão, entre outras características (COSTA, 1999). Logo, abordar questões ambientais, como a conscientização do consumo da água em ambientes educacionais, se mostra como forte contribuinte para o crescimento de uma sociedade mais crítica e ciente da relevância de se preservar os recursos naturais, em específico a água.

$\mathrm{Na}$ literatura é possível identificar algumas iniciativas cujo objetivo é fomentar a importância da preservação da água dentro da escola (MACIEL, 2013). Nesse sentido, em 2013 o Colégio Presbiteriano Quinze de Novembro, da cidade de Garanhuns - PE, vivenciou o projeto Jogo da água: a vida em jogo, que pretendeu promover ações para reflexão sobre o uso desse bem natural. A ação se estendeu a professores e demais funcionários, no entanto, os principais sujeitos foram os estudantes.

O projeto em tela pautou-se pelo seguinte questionamento: a discussão de situações em torno do volume de água gasto diariamente pode otimizar a gestão individual da água? Assim, buscou-se oportunizar situações para reflexão sobre seu uso, por meio de ações que tiveram vários objetivos: a identificação do nível médio diário de gasto de água, a análise do uso cotidiano desse recurso e a adoção de medidas para evitar o desperdício.

Ao considerarmos a importância desses projetos em um ambiente escolar para a formação do indivíduo e conhecendo o contexto tecnológico no qual os estudantes encontram-se atualmente, é perceptível observar que existem recursos tecnológicos como as redes sociais que podem servir de ferramenta de apoio para escola, pois as mesmas promovem interações sociais, trocas constantes de informações entre seus membros, potencialização de competências sociais de interação e comunicação, além de provocar o aprender com o outro (DOTTA, 2010).

Outro elemento que vem se mostrando como uma maneira de motivar os educandos na realização de tarefas cotidianas, é a gamificação, que segundo Lopes et al. (2015), possui a capacidade de criar experiências significativas quando aplicada em contextos da vida diária.

Logo, com base no projeto vivenciado no Colégio Presbiteriano Quinze de Novembro e nas características de rede social e de gamificação, emerge a seguinte questão: "como desenvolver uma rede social que utiliza elementos de jogos para apoiar 
a conscientização do consumo da água?". Diante desse questionamento, o presente artigo busca descrever o processo de desenvolvimento de uma rede social para conscientização acerca do consumo da água, como também tem como objetivo avaliar a rede social com os alunos do Colégio Presbiteriano Quinze de Novembro, visando compreender o que os motivou e o que os fez participar da mesma.

O presente trabalho está organizado da seguinte maneira: a Seção 2 apresenta trabalhos relacionados e o diferencial da proposta apresentada neste artigo; na Seção 3 são apresentados os procedimentos adotados para o desenvolvimento da rede social; a Seção 4 apresenta os resultados do desenvolvimento; a Seção 5 exibe a avaliação e discussão da análise dos dados obtidos a partir do uso da rede social pelos alunos e, por fim, a seção 6 traz algumas considerações finais e aponta trabalhos futuros.

\section{Trabalhos Relacionados}

Esta seção apresenta alguns trabalhos relacionados a este artigo, dentre os trabalhos podemos citar Falcão et al. (2011), que apresentam uma proposta que busca difundir informações na Universidade de Pernambuco através da rede social Twitter, tendo como objetivo usar o potencial dessa rede para aproximar a sociedade local, divulgando ações e projetos. Semelhante ao trabalho de Falcão (2011), a rede social descrita neste artigo tem como objetivo divulgar informações em ambiente educacional, porém relacionadas a recursos sustentáveis, mais especificamente a água.

Peres et al. (2013), apresentam uma proposta que através de uma estrutura tecnológica chamada WAITS ${ }^{1}$ (WAter quality InformaTion System) e das redes sociais das coisas, definida por Presser (2012), e da Internet das coisas, por MacManus (2010), buscam coletar, processar e apresentar informações sobre a qualidade da água e informações ambientais para todos os perfis de usuários, de forma que estas informações sejam utilizadas em pesquisas científicas, ferramentas e atividades de ensino. O tipo de informação do trabalho de Peres (2012), coincide com o que este trabalho busca divulgar, porém o propósito deste trabalho é a conscientização do consumo de água.

Ferreira et al. (2014) relatam o desenvolvimento e avaliação de um jogo educativo que tem como domínio a Educação Ambiental, especificamente Coleta Seletiva e Saneamento Básico. O artigo além de evidenciar a importância da preservação do meio ambiente, evidencia o potencial dos jogos sérios para mediar o processo de ensino e aprendizagem.

Desta forma, o diferencial do presente trabalho diante dos supracitados refere-se à descrição do processo de desenvolvimento de uma rede social gamificada que objetiva contribuir para conscientização do consumo consciente da água, além de sua avaliação com seus potenciais usuários.

\footnotetext{
${ }^{1}$ Provê uma estrutura através de coleta de dados realizada por dispositivos sensores, tratamento e disponibilização em diferentes interfaces. O sistema serve de base para a criação de ferramentas e atividades de ensino e conscientização ambiental.
} 
V Congresso Brasileiro de Informática na Educação (CBIE 2016)

Anais do XXII Workshop de Informática na Escola (WIE 2016)

\section{Processo de Desenvolvimento}

Nesta seção será descrito o procedimento para o desenvolvimento da rede social, denominada Trilha da Água. O procedimento envolveu levantamento de requisitos, gerenciamento, divisão de tarefas, controle de versões e protótipos visuais.

\subsection{Levantamento de requisitos}

Nesta etapa, a partir de reuniões com a coordenadora do Colégio em questão e com os seus alunos, foi possível identificar requisitos funcionais e não funcionais essenciais para o desenvolvimento do software.

Foram pré-estabelecidos os requisitos funcionais apresentadas na Tabela 1. Vale ressaltar que os mesmos se encontram escalados levando em consideração o grau de prioridade: essencial, importante e desejável.

Tabela 1. Requisitos funcionais da "Trilha da Água".

\begin{tabular}{|c|c|l|l|}
\hline Requisito funcional & Essencial & Importante & Desejável \\
\hline Cadastro & $\mathrm{x}$ & & \\
\hline Login & $\mathrm{x}$ & & \\
\hline Postar & $\mathrm{x}$ & & \\
\hline Timeline & $\mathrm{x}$ & & \\
\hline Desafios & $\mathrm{x}$ & & \\
\hline Amigos & $\mathrm{x}$ & & \\
\hline
\end{tabular}

Como requisitos não funcionais, definiu-se a utilização do framework de desenvolvimento web ágil, Django versão 1.7.0. A linguagem de programação Python, para desenvolvimento do backend. A linguagem de marcação HTML5 (HyperText Markup Language) e a linguagem de estilo CSS3 (Cascading Style Sheets) para design do software. Bibliotecas Jquery versão 1.11 .3 e Jquery-ui também foram utilizadas para melhor usabilidade e interatividade do sistema.

\subsection{Gerenciamento}

Para gerenciar as tarefas realizadas no decorrer do desenvolvimento da rede social foi utilizado a aplicação web Trello ${ }^{2}$ que, por sua vez, é destinada a organização de tarefas e eventos. O Trello foi inspirado na metodologia Scrum, processo de desenvolvimento para gerenciar projetos e desenvolvimento ágil de softwares.

\footnotetext{
2 Informações adicionais: <https://trello.com/>
} 
V Congresso Brasileiro de Informática na Educação (CBIE 2016)

Anais do XXII Workshop de Informática na Escola (WIE 2016)

\subsubsection{Divisão de tarefas}

A equipe, para construção e desenvolvimento da rede social, foi constituída de quatro graduandos em Licenciatura em Computação, ficando a divisão das tarefas da seguinte maneira: dois programadores back-end responsáveis em implementar a rede social através da linguagem de programação Python; um programador front-end responsável pela implementação das interfaces de interação com usuário; e um documentador de software.

\subsubsection{Controle de versões}

O controle de versões é de fundamental importância para documentação e acompanhamento da evolução de qualquer sistema. Para tal, utilizou-se o serviço de Web Hosting, GitHub. Este, por sua vez, foi escrito na linguagem Ruby on Rails e possui funcionalidades de uma rede social como feeds, followers, wiki, além de gráficos que sintetizam as contribuições dadas por cada desenvolvedor. Outras funcionalidades interessantes do GitHub são:

- Criação de áreas completamente separadas para testes ou projetos diferentes;

- Desfazer alterações que estão com problemas, voltando para a versão que estava estável;

- Possibilidade de trabalho em equipe de uma forma muito mais simples e segura.

\subsection{Protótipos visuais}

Uma vez definidos os requisitos, foram gerados protótipos por meio da ferramenta de edição gráfica Inkscape ${ }^{3}$ conforme a Figura 1.
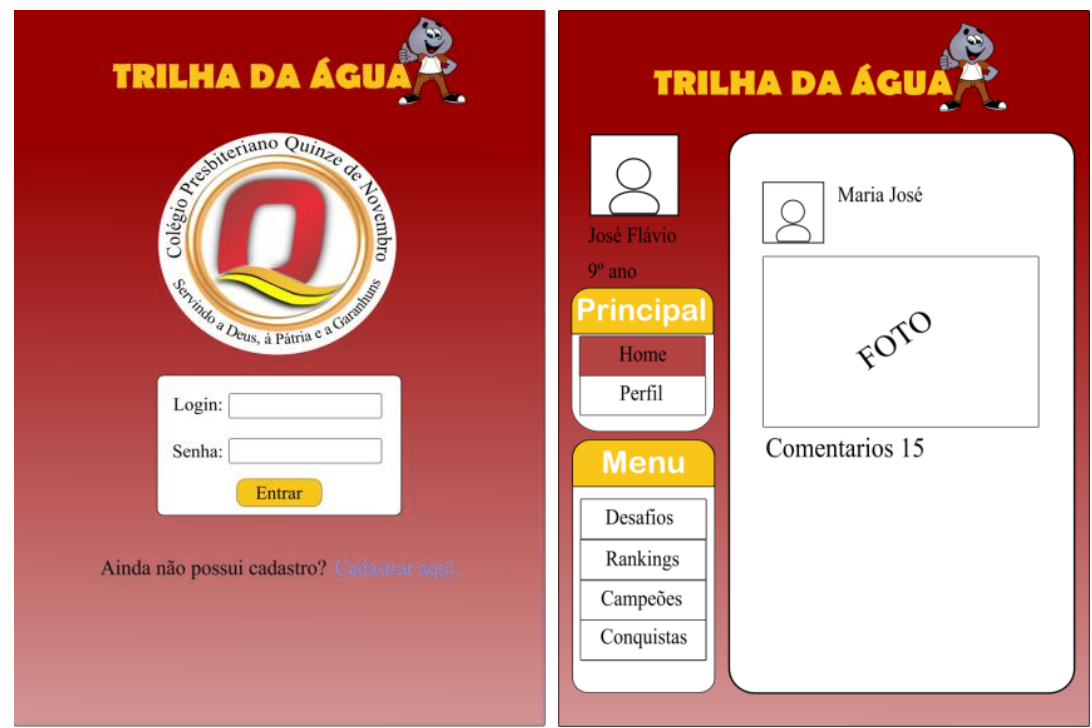

Figura 1. Primeiro protótipo.

\footnotetext{
${ }^{3}$ Informações adicionais: <https://inkscape.org/pt/>.
} 
V Congresso Brasileiro de Informática na Educação (CBIE 2016)

Anais do XXII Workshop de Informática na Escola (WIE 2016)

Na Figura 1, pode-se observar o primeiro protótipo da rede social contendo a interface de login e timeline. Este protótipo busca trazer as principais características, como: cores semelhantes à do colégio e fundamentais funcionalidades do software.

\section{Resultado}

Como resultado, foi desenvolvida uma rede social comtemplada de elementos de jogos. A rede social, denominada Trilha da Água, fornece um ambiente onde alunos, professores e funcionários podem interagir, compartilhar e se desafiarem diante do uso consciente da água.

A Figura 2 sintetiza a tela principal da rede sendo possível identificar suas principais características:

\section{Trilha da Água}

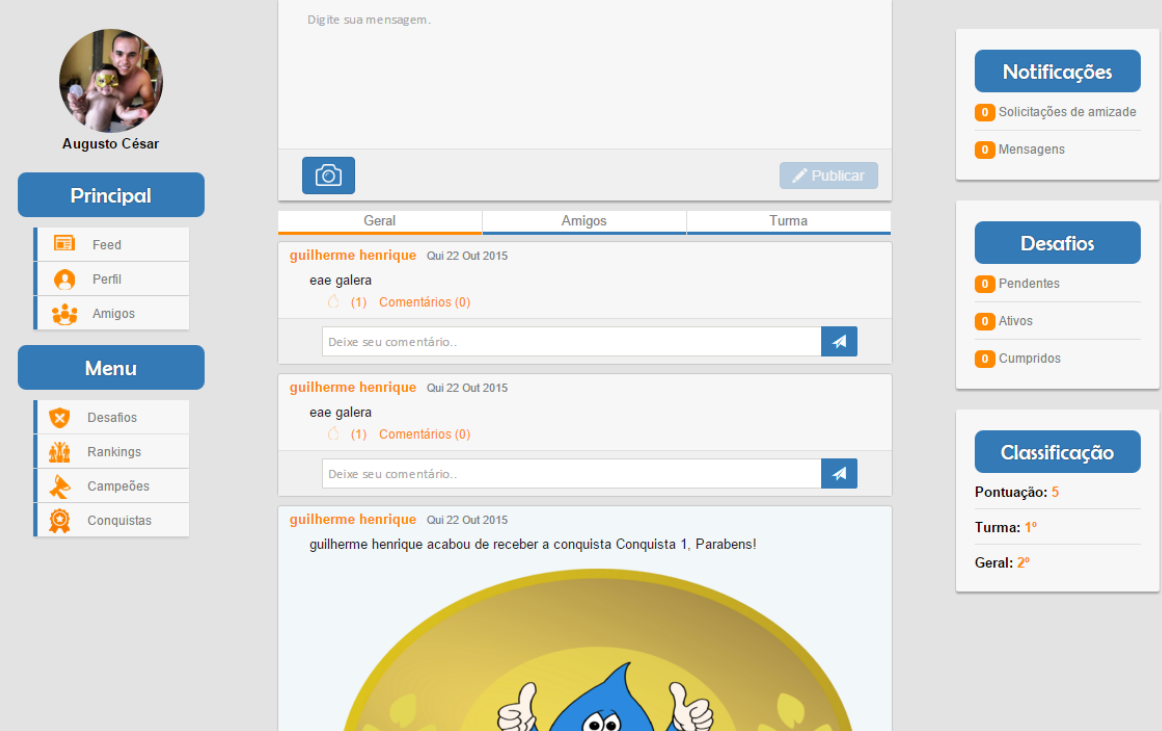

Figura 2. Rede social Trilha da Água.

1. Principal: bloco constituído pela parte social da rede. Nele encontram-se as opções feed, perfil e amigos.

2. Menu: bloco constituído pela parte que engloba a gamificação da rede. Nele encontram-se as opções desafios, rankings, campeões e conquistas.

3. Notificações: bloco responsável por exibir quantas notificações o usuário possui. Nele encontram-se os contadores de solicitações de amizade e mensagens.

4. Desafios: bloco responsável por contar a quantidade de desafios pendentes, ativos e cumpridos que o usuário possui.

5. Classificação: bloco constituído por pontuação, turma e geral. Este bloco é responsável por mostrar de maneira ágil a pontuação atual do usuário e a sua posição em relação à sua turma e toda escola. 
V Congresso Brasileiro de Informática na Educação (CBIE 2016)

Anais do XXII Workshop de Informática na Escola (WIE 2016)

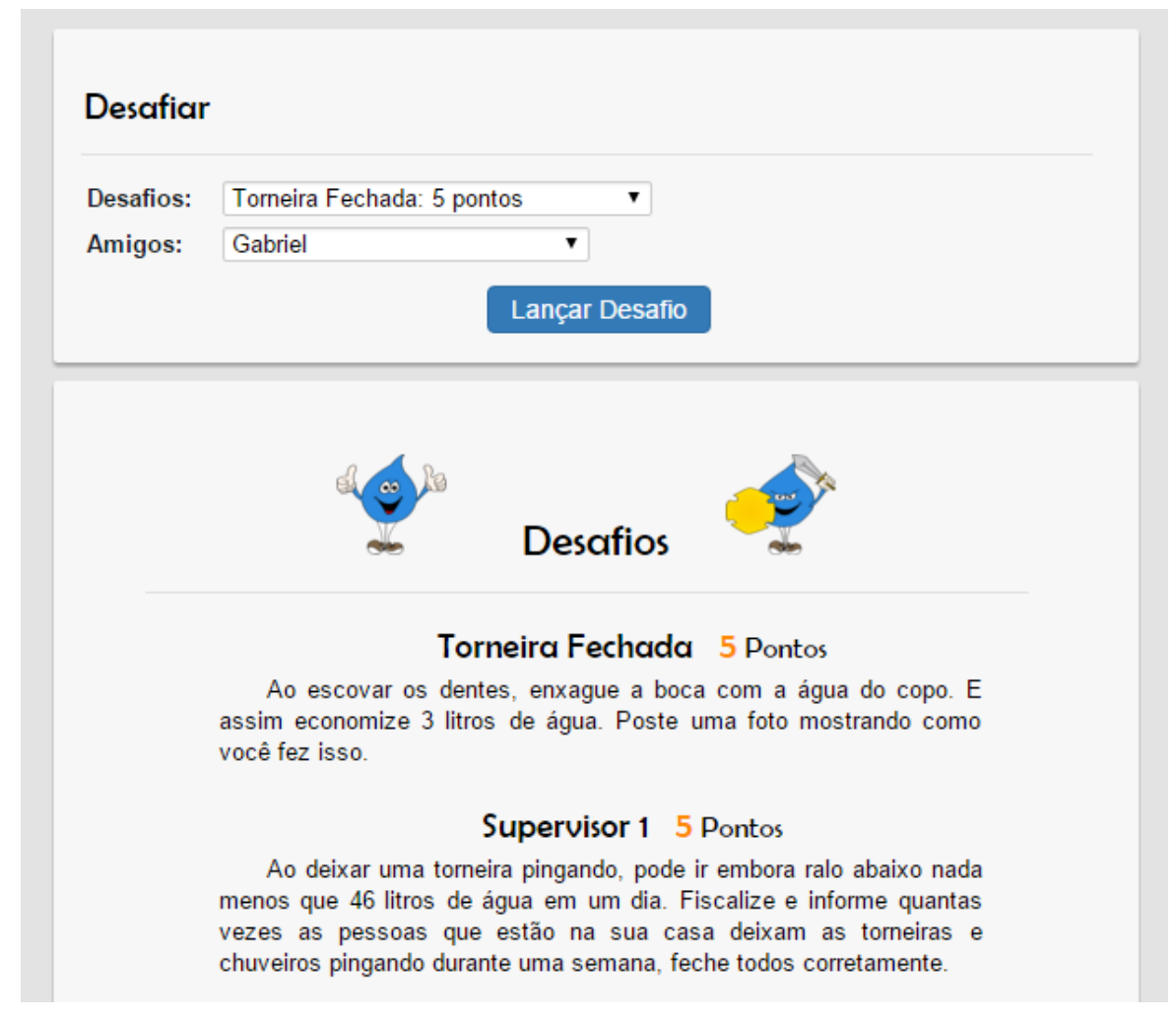

Figura 3. Desafios.

Na Figura 3 é apresentado um cenário caracterizado pela parte gamificada da rede social, no qual é possível visualizar os desafios que os usuários poderão escolher para enviar a outras pessoas na rede social. Todos os desafios são relacionados à temática água e sua preservação, como por exemplo: "ao escovar os dentes, enxague a boca com a água do copo, e assim, economize 3 litros de água. Poste uma foto mostrando como você fez isso". Se o usuário que foi desafiado postar a foto referente ao desafio no feed, o desafiante poderá atribuir uma pontuação para o mesmo (caso o desafio tenha sido cumprido corretamente). A pontuação gerada a partir dos desafios pode ser visualizada na opção ranking no bloco Menu. Na opção campeões também pode ser visualizado qual o usuário que mais pontua durante um mês de uso na rede social.

Outra característica de gamificação na rede social, pode ser visualizada na opção Conquistas, contida no bloco menu, que através do acumulo de pontuações e um determinado número de desafios completados, o aluno libera uma conquista para sua coleção. Por exemplo: Conquista 2, "ganha o aluno que realizar 5 desafios no decorrer de uma semana".

\section{Avaliação da rede social}

A avaliação da rede social foi feita a partir do cadastramento de 88 estudantes dos ensinos Fundamental e Médio do Colégio Presbiteriano Quinze de Novembro da cidade de Garanhuns - PE. A coleta dos dados para avaliação foi feita após um mês de uso da rede social por meio da aplicação de um questionário com 36 estudantes dentre os 88 
V Congresso Brasileiro de Informática na Educação (CBIE 2016)

Anais do XXII Workshop de Informática na Escola (WIE 2016)

cadastrados, número que equivale a aproximadamente $41 \%$ do total de cadastrados (não foi possível aplicar o questionário com todos os estudantes cadastrados, pois houve dificuldade de acesso no momento da coleta dos dados).

Para avaliar "o que fez o usuário ter vontade de usar a rede social" foi definida uma escala onde: Curiosidade representa que o usuário utilizou apenas por curiosidade; Interagir, representa que o usuário utilizou para interagir com colegas e amigos; Jogar, representa que o usuário utilizou para jogar com seus colegas e amigos; conscientizar, representa que o usuário utilizou para conscientização do consumo consciente de água.

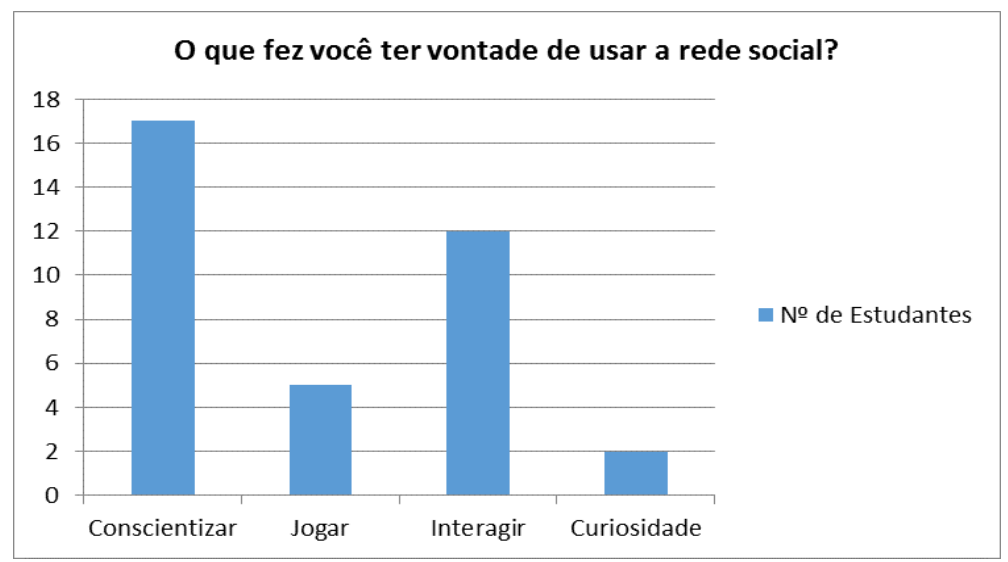

Gráfico 1. Primeira questão.

Logo, foi possível identificar que, 17 estudantes responderam para se conscientizar, 5 estudantes para jogar, 12 estudantes para interagir e 2 estudantes por curiosidade. No Gráfico 1 pode ser observado a distribuição das respostas.

Para avaliar se "a rede social motivou você a economizar água?" foi possível identificar através da escala de Likert (1932), que 12 estudantes concordaram totalmente, 9 estudantes concordaram, 8 estudantes não tiveram opinião, 4 estudantes discordaram e 3 estudantes discordaram totalmente. No Gráfico 2 pode ser observado a distribuição das respostas.

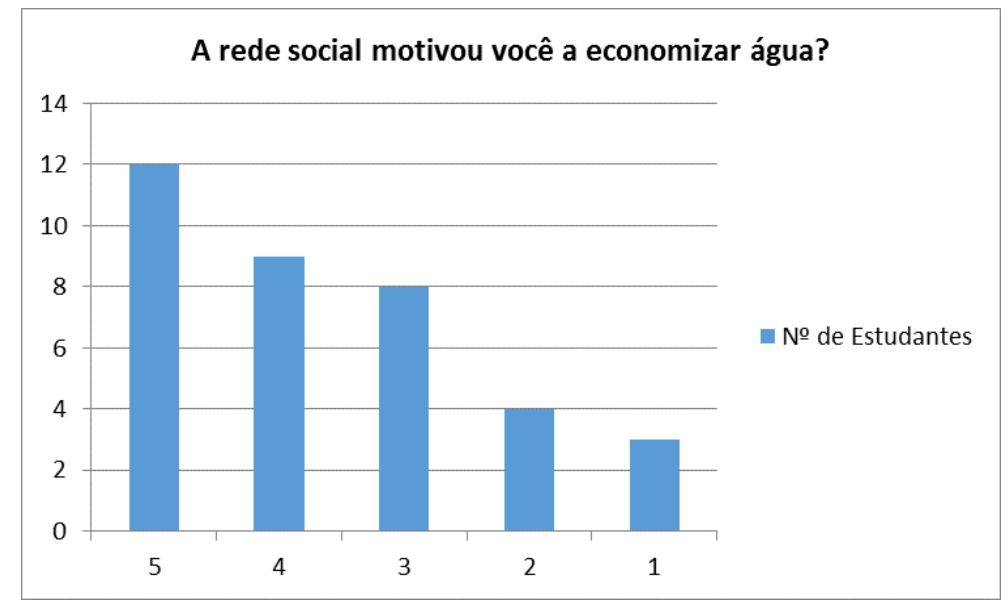

Gráfico 2. Segunda questão. 
Ao analisar estes dados foi possível identificar que aproximadamente $60 \%$ dos alunos concordaram e concordaram totalmente que se sentiram motivados em economizar água através da rede social e cerca de $40 \%$ não opinaram, discordaram e discordaram totalmente em relação a motivação.

Também foi possível analisar que, aproximadamente $47 \%$ dos alunos utilizaram a rede social para conscientizar-se em relação ao consumo da água; Aproximadamente $14 \%$ para jogar; Aproximadamente 33\% para interagir com colegas e amigos e aproximadamente e aproximadamente $6 \%$ por curiosidade.

Desse modo, fica evidente através da análise dos dados que o uso da rede social foi um fator contribuinte para motivar os alunos (o número acima de $50 \%$ demonstra isso) a economizar água e que o espaço fornecido pela rede social possibilitou um ambiente que eles pudessem não só interagir com colegas e amigos, como também economizar água de maneira lúdica.

\section{Considerações finais}

Neste trabalho foi possível descrever o processo de desenvolvimento de uma rede social de acordo com o objetivo proposto no artigo, denominada Trilha da Água, atrelada a elementos de jogos que fosse capaz de apoiar a conscientização do consumo de água no Colégio Presbiteriano Quinze de Novembro da rede particular de ensino, na cidade de Garanhuns - PE.

Também, com base na análise dos resultados do questionário aplicado aos alunos e o objetivo de avaliar a rede social, pôde-se identificar que mais de $50 \%$ dos alunos concordaram e concordaram totalmente que se sentiram motivados em economizar água através da rede social. Além disso, foi compreendido que a maior vontade dos estudantes em utilizar a rede social, foi de conscientizar-se em relação ao consumo de água e interagir com colegas e amigos.

Como trabalhos futuros, pretende-se aplicar e avaliar a rede social em outras escolas, de preferência da rede pública de ensino, visando comparar os dados obtidos neste artigo com dados coletados em outras escolas. Além disso, pretende-se incluir outras questões ambientais, tais como: desmatamento e combate ao mosquito aedes aegypti. Desse modo, ampliando, difundido e fortalecendo projetos na área de educação ambiental de maneira interativa, motivadora e colaborativa.

\section{Referências}

Costa, J., (1999). O papel da escola na sociedade actual: implicações no ensino das ciências. Millenium. Disponível em: < http://repositorio.ipv.pt/bitstream/10400.19/871/1/O\%20papel\%20da\%20escola\%20 na\%20sociedade\%20actual.pdf>. Acesso em: 29/05/2016.

Falcão L. C. T., Junior C. G. S., Arruda. F. T., (2011). "Proposta de uso do Twitter como ferramenta de informação e conhecimento na Universidade de Pernambuco". In: Anais do $22^{\circ}$ Simpósio Brasileiro de Informática na Educação (SBIE 2011), Aracaju-SE, p. 952-955 de Novembro de 2011. 
V Congresso Brasileiro de Informática na Educação (CBIE 2016)

Anais do XXII Workshop de Informática na Escola (WIE 2016)

Ferreira M. A., Santos. H. M., Lucena. A. M., (2014). "NiceTown - Um Jogo Eletrônico para Ensino da Educação Ambiental". In: Anais do Simpósio Brasileiro de Informática na Educação (CBIE 2014), p. 298- 307.

JACQUIMINOUT, J. J. P., (2015). "Sistema de Reaproveitamento de Água Residuais de Processos Industrial e Esgoto Residenciais por meio de Transformação Térmicas Físico Química”. Disponível em: < http://qib.bz/3p/>. Acesso em: 02/06/2016.

LIKERT, R., (1932). "A technique for the measurement of attitudes. Archives in Psychology", 140, p. 1-55.

Lopes, R. A.; Toda, A. M.; Brancher, J. D., (2015) Um estudo preliminar sobre elementos extrínsecos e instrínsecos do processo de Gamification. In: Revista Brasileira de Informática na Educação RBIE, v. 23, n. 3, pág. 165-173.

Peres A., Miletto E. M., Kapusta. S., Ojeda. T., (2013). "Monitoramento da Qualidade da Água, Redes Sociais das Coisas e a Educação Ambiental”. In: Anais do $2^{\circ}$ Congresso Brasileiro de Informática na Educação (CBIE 2013), p. 2- 11.

S. Dotta, (2010). "Uso de uma Mídia Social como Ambiente Virtual de Aprendizagem". In: Anais do $22^{\circ}$ Simpósio Brasileiro de Informática na Educação - $17^{\circ}$ Workshop de Informática na Escola. - Aracaju-SE, 2010, URL http://www.academia.edu/1290792/Uso_de_uma_Midia_Social_como_Ambiente_Vi rtual_de_Aprendizagem.

UNESCO, (2013). “Água, fonte de vida: cooperação pela água”. Disponível em: $<$ http://www.unesco.org/new/pt/brasilia/about-this-office/prizes-andcelebrations/2005-2015-international-decade-for-action-water-for-life/>. Acesso em: 29/05/2016.

Maciel. S. M. A., Everton, S. F., (2013). “O Uso e Reuso da Água nas Escolas Municipais Rurais de Sant'Ana do Livramento: Importantes Dimensões para o Desenvolvimento de Políticas Públicas”. In: Anais XXXVII Encontro da ANPAD, Rio de Janeiro - RJ. 